expectations, reduced significantly in many countries. Indeed, the numbers of new diagnoses has increased in some European countries, the USA and Australia. In the UK, for example, over 3000 MSM were diagnosed with HIV in 2010, the highest number since the beginning of the epidemic. As a consequence, the prevalence of HIV is increasing and, for gay communities, a growing number of men are now living with HIV.

Although incidence of sexually transmitted infections (STIs) in MSM fell dramatically in the early years of AIDS, post-ART we have seen a return in some jurisdictions to pre-AIDS incident STIs, and the emergence of new sexually transmitted pathogens.

This paper will focus on behavioural and epidemiological research in the era of ART, with particular reference to the continuing transmission of HIV. It will review current risk factors for HIV infection in negative MSM, sexual risk behaviour and risk reduction interventions among HIV positive MSM, and the emergence and re-emergence of new and established STIs. It will conclude with current challenges to the prevention of HIV and STIs in MSM, and prospects for the future.

\section{S13.2 EVIDENCE-BASED TARGETED REVIEW OF HIV AND STI PREVENTION INTERVENTIONS AMONG MSM}

doi:10.1136/sextrans-2013-051184.0062

\begin{abstract}
'A Thorson, 'S Strömdahl, ${ }^{2} \mathrm{M}$ Sabido, ${ }^{3}$ A Pharris. 'Karolinska Institute, Stockholm, Sweden; 'Fundació Sida i Societa, Barcelona, Spain; ${ }^{3}$ European Centre for Disease prevention and Control, Stockholm, Sweden
\end{abstract}

Background In Europe, men who have sex with men (MSM) are disproportionately affected by STI/HIV. Sex between men is the predominant mode of HIV and syphilis transmission and contributes to a third of gonorrhoea cases in 2011. The main goal of this evidencebased review was to gather and critically appraise the scientific evidence for and to inform European guidance on a comprehensive approach for HIV, STI and hepatitis prevention among MSM.

Methods Relevant data bases were searched for publications related to disease prevention and health promotion among MSM. All kinds of reviews were included but gave presence to systematic reviews, when no reviews were found individual studies were included. A critical appraisal of the quality of each study was performed before inclusion in the targeted review. The Highest Attainable Standard of Evidence (HASTE) was used for grading as the basis for recommendations as HASTE was specifically developed to evaluate evidence regarding HIV/STI interventions among key populations. HASTE takes into account three categories with equal weight: efficacy data; implementation science data; biological and public health plausibility. Results Seven interventions were identified to be strongly recommended for prevention of HIV, STI and/or hepatitis, and 15 interventions for probable or possible recommendation. Consistent condom use, HIV treatment as prevention, peer outreach and support groups for men testing HIV negative as well as for HIV positive men, together with HSV-2 suppression therapy with acyclovir to prevent genital ulcer disease and hepatitis B vaccination, were all strongly recommended according to the HASTE grading.

Conclusions In general, studies of acceptable quality from the European context were few and made the assessment of context-specific effectiveness difficult. Additionally, effectiveness studies looking into real-life effectiveness of biomedical interventions were very few. Future research would be able to contribute with more information from the specific European settings including (cost-) effectiveness studies.

\section{S13.3 THE STATUS OF HIV PREVENTION AMONG MSM: AN OVERVIEW OF THE EUROPEAN RESPONSE}

doi:10.1136/sextrans-2013-051184.0063

O Sfetcu, T Noori, G Spiteri, A Pharris, M van de Laar. ECDC, European Centre for Disease Prevention and Control, Stockholm, Sweden
Introduction MSM in the European Union and European Economic Area (EU/EEA) are disproportionately affected by STI: 39\% of all HIV diagnoses, $33 \%$ of gonorrhoea and $55 \%$ of syphilis cases were reported among MSM in 2011.

Methods Analyses of surveillance data and information from countries responses for Dublin Declaration monitoring were combined with a review of existing national prevention intervention programmes targeted at MSM. Characteristics of 118 prevention interventions studies were included in the repository.

Results Outbreaks of syphilis, hepatitis $C$ and lymphogranuloma venereum and increasing trends of gonorrhoea and HIV among MSM, observed between 1995-2011, were reported to be associated with high levels of risk behaviour, sexual networking and socioeconomic and cultural factors. The national responses included: strenghtening of surveillance, prevention and care; enhanced partner notification; and development of a range of prevention intervention programmes. The majority of prevention interventions used media campaigns, education and counselling followed by harm reduction strategies like condom distribution and HIV/STI testing alone or combined with other activities. Nearly half of the behavioural interventions studies reported proper outcome evaluation.

In 2012, HIV/STI prevention programmes targeted to MSM were implemented in 22/30 EU/EEA countries, with non-governmental organisations playing a key role in programme implementation through campaigns, outreach work, information provision and condom distribution. Reported coverage of HIV prevention programmes for MSM ranged from $43 \%$ to $76 \%$ across countries. Rates of HIV testing among MSM during the last 12 month ranged from $12 \%$ to $74 \%$ and rates of condom use at last anal intercourse from $42 \%$ to $64 \%$.

Conclusion Diversity across EU/EEA in the design and implementation of prevention intervention strategies reflects various characteristics of MSM populations and the changing epidemiology of STI/HIV. A common need exists for improving the effectiveness of prevention intervention programmes, for targeting young, migrant and ethnic-minority MSM and for ensuring adequate funding.

\section{S13.4 WHAT DO GAY MEN NEED? - CHALLENGES REGARDING COMPREHENSIVE APPROACHES IN HIV-PREVENTION INTERVENTIONS FROM A NATIONAL PERSPECTIVE}

doi:10.1136/sextrans-2013-051184.0064

D Sander. Deutsche AIDS Hilfe, Berlin, Germany

Regarding empirical evidence gay men are facing several health problems which can arise from the mastering of living in homonegative environment. We can e.g. find high levels of substance use, mental health problems, and risky behaviour.

The presentation will raise the question on how these different and interacting health matters work together and how they can be tackled in campaigns focused on gay men and other MSM. What is the experience of the national German campaign "I KNOW WHAT I'M DOING"; what can be done in future work from the MSM community perspective; who are the necessary co-operative partners, what has to be requested from different policy sectors?

\section{S.14 - New strategies and implementation of best practises for dual elimination of mother-to-child transmission of syphilis and HIV (organised by WHO)}

\section{S14.1 TESTING FOR SYPHILIS IN PREGNANCY AND ASSOCIATED ADVERSE OUTCOMES IN MOZAMBIQUE}

doi:10.1136/sextrans-2013-051184.0065 
${ }^{1} M$ Mahomed, ${ }^{2,3}$ S Gimbel, 4,5R Hoek, ${ }^{6} \mathrm{~A}$ Rustagi, ${ }^{7} \mathrm{C}$ Come, ${ }^{8} \mathrm{~L}$ Newman, ${ }^{2} \mathrm{~F}$ Faria, 7.5 Manuel, ${ }^{2,3}$ S Gloyd, ${ }^{9} \mathrm{~N}$ Broutet. 'Instituto Nacional de Saúde (INS), Maputo, Mozambique; ${ }^{2}$ Health Alliance International, Seattle, WA, United States; ${ }^{3}$ University of Washington, Washington, WA, United States; 'University of Washington, Seattle, WA, United States; ${ }^{5}$ Centro de Investigação Operacional da Beira-INS, Beira, Portugal; ${ }^{6}$ Centro de Investigação Operacional da Beira-INS, Covilhã, Portugal; ${ }^{7}$ National Institute of Health, Maputo, Mozambique; ${ }^{8}$ World Health Organization, Geneva, Switzerland; ${ }^{9}$ World Health Organization, Geneva, Switzerland

Background Antenatal syphilis screening and treatment is an effective intervention to reduce perinatal, infant, and maternal morbidity and mortality. Prenatal syphilis screening increased in Manica and Sofala Provinces from < 5\% in 1993 to over $90 \%$ since 2005. This study aimed to (1) estimate the prevalence of congenital syphilis in the cities of Beira and Chimoio, (2) determine differences in congenital syphilis among women treated early in pregnancy, late in pregnancy, and not treated, and (3) identify factors associated with syphilis screening among pregnant women.

Methods Pregnant women presenting at one of six maternities in Beira and Chimoio cities, were recruited and screened with rapid syphilis tests (RST) and, if positive, further screened using Rapid Plasma Reagin (RPR). All live newborns whose mothers tested positive at any point during pregnancy or at birth were RPR screened to detect congenital syphilis. A newborn subsample also had blood and tissue samples taken for IgM and PCR testing to validate the RPR results.

Results Between March 5, 2012 and March 15, 2013, 16,812 women were recruited out of the 19,821 births registered at the participating maternities. Overall, 611 women (3.6\% total) tested positive for syphilis; 498 were syphilis positive by RST at the maternity. An additional 113 women had tested positive at ANC, but were RST negative at delivery. Of the total 405 women who had tested syphilis positive (RST or RPR) and been treated in ANC, their syphilis results at delivery were the following: 286 (71\%) were RST positive, 192 (47\%) were both RST and RPR positive, and 35 (9\%) were RPR positive and RST negative. There was no significant difference in RST/RPR rates between women treated before or after 28 weeks gestation. Of the 16,322 women who were syphilis negative at ANC, 211 (1.26\%) were RPR positive at birth (suggestive of recent infection); of these women, 152 (54\%) were RPR positive. Estimates of the prevalence of and factors associated with congenital syphilis and association with gestational age of treatment are pending, awaiting results of confirmatory testing.

Conclusions The congenital syphilis prevalence rate is substantially lower in this region than in other regions of Mozambique, likely related to years of antenatal syphilis screening. Lifetime positivity of RST was variable, as nearly $30 \%$ of women with positive RST tests in pregnancy were RST negative at birth. In addition, nearly half of women treated adequately remained RPR positive at delivery after treatment.

\section{S14.2 THE CISNE PROJECT: IMPLEMENTATION OF POCT FOR SYPHILIS AND HIV IN ANTENATAL CARE AND REPRODUCTIVE HEALTH SERVICES IN PERU}

\section{doi:10.1136/sextrans-2013-051184.0066}

'P Garcia, ${ }^{2} \mathrm{R}$ Peeling, ${ }^{3} \mathrm{D}$ Mabey, ${ }^{4} \mathrm{~K}$ Holmes, ${ }^{5} \mathrm{C}$ team. ${ }^{1}$ Universidad Peruana Cayetano Heredia, Lima, Peru; 'London School of Hygiene and Tropical Medicine, London, UK; ${ }^{3}$ London School of Hygiene and Tropical Medicine, London, UK; ${ }^{4}$ University of Washington, Seattle, WA, United States, ${ }^{5}$ CISNE team, Lima, Peru

Background Congenital syphilis and syphilis in pregnancy in Peru persist as important public health issues, and improvement of screening/treatment for pregnant women remains challenging. Rapid syphilis testing (RST) allows simple and immediate diagnosis and treatment at a single clinic visit and could increase screening and treatment coverage reducing the incidence of stillbirth and congenital syphilis and generate in the long term a sustainable cost effective intervention.
Methods We tested the feasibility, performance, impact and costeffectiveness of implementing RST in an underserved urban area at a biggest maternity hospital in Peru and a network of 16 peripheral health centres offering prenatal care in a periurban poor area in $\mathrm{Cal}-$ lao-Ventanilla, Peru. RST (integrated with HIV rapid test: the "two for one") were offered at the first prenatal visit (ANC), at delivery and within miscarriage/abortion services.

Results Data from the baseline pre-implementation evaluation revealed limited coverage of screening and treatment services for maternal syphilis and a complex and inefficient system for ANC. RST was started in January 2010. Overall success of implementation was measured by rates of maternal syphilis screening and treatment coverage, partner treatment, and acceptability of RST among providers and patients. We also performed a cost-effectiveness analysis of RST against the Rapid Plasma Reagin (RPR). Attention was paid to the process of dissemination and transfer activities to the Ministry of Health of Peru, through the involvement of both the National Program of STIs and HIV and the Reproductive Health Program. National guidelines have been modified, and recommend the use of both tests, RST and rapid HIV testing in the screening of pregnant women.

Conclusions RST implementation was feasible, successful, acceptable and cost effective. Its introduction catalysed improvements in the quality of care, and by the end of the project it has been introduced in the country as a National policy.

\section{S14.3 EXPLAINING INACTION: THE POLITICS OF CONGENITAL SYPHILIS AND THE GLOBAL HEALTH AGENDA}

doi:10.1136/sextrans-2013-051184.0067

1' Bump, ${ }^{2} \mathrm{~N}$ Salisbury. 'Georgetown University, Washington, DC, United States; ${ }^{2} \mathrm{PATH}$, Seattle, WA, United States

Background If disease burden were directly correlated with priority on the global health agenda, then congenital syphilis would have a high position. But even though congenital syphilis adversely affects around 650,000 pregnancies and newborns annually and is relatively simple to diagnose and treat, it is largely absent from high-level discussions of reproductive and child health and is not a major programmatic focus at prominent global health organisations. We sought to examine explanations for the low prioritisation of congenital syphilis and use our findings to inform framing strategies to improve the placement of congenital syphilis on the global health agenda.

Methods First, we reviewed published literature on congenital syphilis to construct a basic narrative, identify trends, and identify important stakeholders. Second, we conducted semi-structured interviews with stakeholders involved with or important to congenital syphilis control to understand the prioritisation of congenital syphilis and attempts to promote it. Third, we surveyed influential representatives of global health institutions to assess their perspectives on the position of congenital syphilis on the global health agenda. Finally, we analysed the data to track changes in priority given to congenital syphilis over time. We then developed political strategies based on these data.

Results Based on our literature review and key informant interviews, we develop framing strategies, identify potential political allies, and suggest pathways advocates can use to increase the prominence of congenital syphilis on the global health agenda.

Conclusions Although it is an important cause of stillbirth and neonatal death, congenital syphilis is largely invisible on the global health agenda. Past efforts to raise awareness have had limited impact. Future efforts toward elimination of congenital syphilis could benefit greatly from increased attention to framing strategies and alliances with related issues in global health. 